\title{
Resultados en Saber Pro de estudiantes de modalidad presencial y virtual en dos universidades colombianas ${ }^{1}$
}

\author{
Deisy Ortiz Romero² ${ }^{2}$ Erika Gómez Villarreal ${ }^{3} \&$ Nicolás Arias Velandia $^{4}$ \\ Institución Universitaria Politécnico Grancolombiano, Colombia
}

Recibido, junio 19 de 2015

Concepto evaluación, septiembre 14 de 2015

Aceptado, octubre 15 de 2015
Referencia: Ortiz Romero, D.; Gómez Villarreal, E.; Arias Velandia, N. (2015). "Resultados en Saber Pro de estudiantes de modalidad presencial y virtual en dos universidades colombianas". Revista Academia y Virtualidad, 8, (2), 100-111

\section{Resumen}

En este trabajo se analizan las diferencias en los resultados en las pruebas Saber Pro de egresados de administración de empresas de modalidad presencial y virtual de dos instituciones colombianas de Educación Superior. Las dos instituciones comparadas se ubican en promedios históricos cercanos en la prueba Saber Pro del ICFES. Se descargaron bases de datos de los resultados de las pruebas Saber Pro de ambas instituciones del sistema FTP-ICFES y se operó con los puntajes numéricos, los nombres de las instituciones y la modalidad o metodología virtual o presencial de sus estudiantes, de los estudiantes de administración de empresas de ambas instituciones. Se evidenciaron más diferencias entre instituciones que entre modalidades en los resultados de los egresados.

Palabras clave: rendimiento comparado, evaluación, aula (ERIC European Education Thesaurus), aprendizaje en línea (Unesco Thesaurus).

1. Artículo resultado del trabajo de grado de las dos primeras autoras, producto del semillero de investigación "Logro Educativo en estudiantes de programas de Educación Superior presencial y virtual”, adscrito al proyecto de investigación Diferencias de logro educativo e inclusión laboral en egresados de programas presenciales y virtuales de Educación Superior, aprobado y en financiación en la Convocatoria de Investigación Aplicada 2013 (IA2013) de la Institución Universitaria Politécnico Grancolombiano (código 2014-FCS-PEC-TC-436). Grupo de Investigación Psicología, Educación y Cultura / Observatorio de Educación, Institución Universitaria Politécnico Grancolombiano.

2. Departamento Académico de Psicología, Facultad de Ciencias Sociales, Institución Universitaria Politécnico Grancolombiano. Psicóloga en formación. Estudiante de práctica profesional; Integrante Semillero de Investigación "Logro Educativo en estudiantes de programas de Educación Superior presencial y virtual". Correo: dey.ortiz@hotmail.com

3. Departamento Académico de Psicología, Facultad de Ciencias Sociales, Institución Universitaria Politécnico Grancolombiano. Psicóloga en formación. Estudiante de práctica profesional; Integrante Semillero de Investigación "Logro Educativo en estudiantes de programas de Educación Superior presencial y virtual". Correo: tata010@hotmail.com

4. Líder Observatorio de Educación, Departamento de Investigación, Desarrollo e Innovación. Institución Universitaria Politécnico Grancolombiano. Magíster en Educación, Universidad Pedagógica Nacional. Correo: nariasv@poligran.edu.co 


\title{
Saber Pro results. Face-to-face and online students at two Colombian universities
}

\begin{abstract}
This paper analyzes differences of Saber Pro test results regarding face-to-face and virtual types of business administration graduates at two Colombian higher education institutions that recorded close historical scores of Saber Pro test ICFES. Results also were downloaded using FTP-ICFES database system, and analyses were run using subtest scores, institution names, modes or methodology (virtual or face-to-face) by business administration graduates of both institutions considered. Results showed more significant differences between institutions rather than modes.
\end{abstract}

Keywords: comparative achievement, assessment, classroom (ERIC European Education Thesaurus), online learning (Unesco Thesaurus).

\section{Resultados no Saber Pro de estudantes de modalidade presencial e virtual em duas universidades colombianas}

\section{Resumo}

Neste trabalho analisam-se as diferenças nos resultados nas probas Saber Pro de graduados em administração de empresas de modalidade presencial e virtual de duas instituições colombianas de Educação Superior. As duas instituições comparadas situam-se em médias históricas contiguas nas probas Saber Pro do ICFES. Descarregaramse bases de dados dos resultados das provas Saber Pro de ambas instituições do sistema FTP-ICFES e se operou com escores numéricos, os nomes das instituições e a modalidade ou metodologia virtual ou presencial dos seus estudantes, dos estudantes de administração de empresas de ambas instituições. Evidenciaram-se mais diferenças entre as instituições que entre modalidades nos resultados dos graduados.

Palavras chave: rendimento comparado, avaliação, aula (ERIC European Education Thesaurus), aprendizagem em on-line (Unesco Thesaurus).

\section{Introducción}

Son múltiples y variados los factores que inciden en el desempeño de un estudiante. Una manera de definirlo es a partir del concepto de logro educativo. El logro educativo, según Moreno (1998), es el aprendizaje producto de la exposición al sistema educativo o a una forma determinada de educación. Dentro de esta categoría, se comprenden además distintas dimensiones como la construcción de conocimientos, el desarrollo de habilidades, la formación de hábitos y actitudes y la internalización de valores, entre otros. Para Hederich y Camargo (2000), el logro educativo es una variable de naturaleza individual y su medida se determina a través de un juicio de valor acerca de lo alcanzado por el estudiante conforme a criterios específicos de logros mínimos que deben ser alcanzados en cada momento de su trayectoria dentro del sistema educativo.

En este sentido, las pruebas externas constituyen una medida externa del logro educativo de los estudiantes. En 
el caso de Colombia, las pruebas Saber Pro son exámenes que presentan los estudiantes al finalizar su formación en instituciones de Educación Superior. En esta pruebas se evalúan competencias genéricas -aplicadas para estudiantes de todas las formaciones, que incluye las áreas de lectura crítica, razonamiento cuantitativo, composición escrita, inglés y competencias ciudadanasy competencias específicas -que se evalúan solamente en estudiantes pertenecientes a programas de una misma área de conocimientos, e.g, pruebas de competencias específicas para estudiantes de carreras de ingeniería, o pruebas de competencias específicas para estudiantes en carreras de ciencias sociales (Abuchar \& Simanca, 2013).

En este contexto, surgen inquietudes con respecto a si se presentan diferencias de desempeño entre estudiantes en diferentes modalidades y ofertas de Educación Superior en Colombia. En este sentido, Lapsley, Kulik, Moody y Arbaught (2008) sugieren que existe una comprensión similar de los estudiantes tanto que toman clases de manera virtual, como los que asumen las clases del modo tradicional. Para este estudio, se dividieron los estudiantes en dos secciones, con la característica que ambos los dirigía el mismo profesor y estaban cursando el mismo semestre. Sin embargo, los dos grupos diferían, evidentemente, en el formato de presentación de sus clases.

Uno de ellos, asistía al salón de clases del modo tradicional, en los cuales algunos ejercicios se presentaban en línea, mientras que el otro grupo presentaba en línea todos los ejercicios, lecturas y tareas. Los autores de este artículo se basan en una teoría de Simonson, Schlosser y Hanson (1999), que sugiere debe haber una equivalencia en las experiencias que tienen ambos tipos de educación para que, asimismo, el logro educativo sea equivalente. La metodología fue enviarle el mismo material a los grupos de estudiantes sobre "El manejo de los recursos humanos".

En ambos grupos hubo discusión de los capítulos del libro que contenían estos temas: un grupo lo hizo a través de un foro virtual y el otro durante la clase presencial. Del mismo modo, el profesor no podía verse con los estudiantes en línea cara a cara en ningún momento. En particular se tomó de cada estudiante tres variables para tener en consideración, las cuales se midieron al comienzo de la investigación: el promedio de las calificaciones, los créditos obtenidos a la fecha y la edad.

A los estudiantes de ambos grupos se les pidió que realizaran 16 evaluaciones, cada uno de ellos compuesta por 15 preguntas de selección múltiple. Ambos grupos presentaron la evaluación en las mismas condiciones, es decir, con el mismo tiempo para responder las preguntas se realizaron en el mismo orden, entre otros. En este estudio los estudiantes del curso en línea obtuvieron mejores resultados. Esto parece suceder debido a que éstos están más acostumbrados que los estudiantes presenciales a realizar tareas en formato electrónico.

De igual manera, se han realizado investigaciones en las que se considera relevante el componente cognitivo y motivacional de los estudiantes a la hora de las calificaciones y la influencia de estos componentes en ambas modalidades. Se establece que para la obtención del éxito académico los alumnos precisan en la voluntad y la habilidad (Suárez y Anaya, 2004). Según Suarez y Anaya (2004), en la educación de modalidad presencial los estudiantes desarrollan mayores estrategias de aprendizaje con otros compañeros, pero también desarrollan mayores niveles de ansiedad. En cambio en la educación a distancia se encuentra el mayor nivel motivacional debido a la mayor valoración de tareas, un nivel de autosuficiencia más alta y mayor percepción de control.

Se evidencia entonces, de acuerdo con la literatura investigativa, que las comparaciones del logro educativo en estudiantes de modalidad presencial y virtual suele mostrar dos tipos de resultados: la presencia o la ausencia de diferencias en dicho logro. Un primer conjunto de investigaciones no encontró diferencias estadísticamente significativas entre los logros evidenciados por grupos de estudiantes de cursos virtuales y tradicionales sobre el mismo contenido (Coma, 2006; Kirtman, 2009; Carrol \& Burke, 2010; Gibson, 2008; Brownstein \& Gerlowski, 2008; Larson \& Sung, 2009; Tutty \& Kleine, 2008), casi independientemente del área y de la medida de logro utilizada en dichos estudios.

En el segundo caso se reportan evidencias de mayor logro en estudiantes de modalidad presencial o mayor 
logro en estudiantes de modalidad virtual. El conjunto de investigaciones que muestra mayor logro de los estudiantes en modalidad virtual encontraron que esta ventaja sobre los estudiantes presenciales se relaciona con:

- Un mayor seguimiento del profesor sobre el desempeño de sus estudiantes (Shutte, 1997; Wong \& Tantall, 2009).

- La posibilidad que dan las herramientas informáticas al estudiante para regular su tiempo y sus recursos, sin necesidad de someterse a dictados externos de horario o lugar de estudio (Coma et al, 2006).

- Hacer que los estudiantes tengan más interacciones con el formato gráfico y con los textos, que les permite volver sobre el material y tener una idea más integral del contenido (Smith et al, 2001).

- Dejar interactuar sincrónica y asincrónicamente al estudiante con el profesor y al estudiante con otros estudiantes (Onrubia, 2005; Smith et al, 2001)

Por otra parte, otro conjunto de investigaciones muestra mayores logros de los estudiantes de modalidad presencial, y lo atribuyen a que la educación en modalidad virtual y con el uso de objetos virtuales en el aprendizaje favorece algunos rasgos o estilos de actuación que solamente comparten algunos estudiantes: aquellos con tendencias de procesamiento de información más analíticas que holísticas (López, 2010; López \& Triana, 2013; Smith et al., 2001) y a que sería frecuente que los estudiantes enrolados voluntariamente en cursos virtuales son solamente un subconjunto de estudiantes más motivados por tener mayor familiarización o conocimientos más avanzados sobre los contenidos abordados (McGovern, 2004; Prensky, 2001) y que dichos rasgos no se pueden atribuir a todos los estudiantes.

Por otra parte, estos trabajos debaten que la educación presencial posee elementos de interacción social y escucha, que favorecen más a los estudiantes que valoran más dichos elementos (Camargo, 2013; Camargo \& Hederich, 2007) y que en ciertas implementaciones concretas de modalidades virtuales se suelen sacrificar
(Silvio, 2004; Lugo, Vera-Rossi \& Flood, 2004; Padilla Omiste, 2004; Vianney, Torres \& Farias, 2004; Rivera Rivera, 2004; Turpo, 2012).

No se puede establecer, entonces, a partir de los estudios una única tendencia en el logro educativo. En su reporte, Arias-Velandia, Hederich y Guarnizo (2014) muestran que es más frecuente no encontrar diferencias de logro entre los estudiantes de modalidad presencial o virtual, pero esta tendencia sólo tiene una investigación más en su favor que el hallazgo de mayores logros en educación virtual y tres más que el hallazgo de mayores logros en educación presencial (figura1).

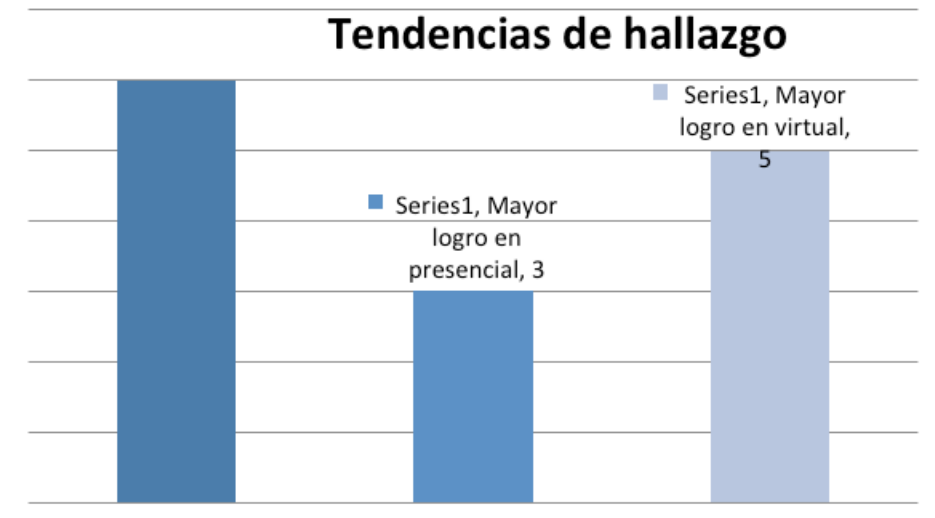

Figura 1. Tendencias de los hallazgos en los reportes de investigación analizados. Fuente: Arias-Velandia, Hederich \& Guarnizo, 2014.

Sin embargo, en un estudio más reciente, Arias-Velandia, Hederich y Trujillo-Flórez (2015) encuentran que cerca de $66 \%$ de los estudios no muestran diferencias significativas entre los estudiantes en modalidad presencial y virtual y que la mayoría de estos estudios plantean medidas internas de evaluación del profesor, en comparación con muy pocos estudios que utilizan pruebas censales o pruebas estandarizadas como criterio de evaluación externa del logro.

Por otra parte, Rodríguez Albor, Gómez Lorduy y Ariza Dau (2014) encuentran, al comparar estudiantes en modalidad presencial y virtual de dos universidades colombianas en su desempeño en la prueba Saber Pro que es de carácter censal y es aplicada por un ente externo a las instituciones, el Sistema Nacional de Evaluación de Calidad de la Educación ICFES- una tendencia de 
mayores logros en estudiantes de modalidad presencial en programas de psicología y de licenciaturas en educación, pero reconocen que no pudieron desligar eficientemente la variable modalidad (presencial o virtual) de otras como el modelo pedagógico, que a veces parecía diferir también entre modalidades.

Las anteriores investigaciones sobre logro en Educación Superior presencial y virtual permiten plantear lo siguiente:

1. A pesar del avance en el campo, existen dudas de cuáles factores realmente pueden incidir en que haya o no haya diferencias entre modalidades presenciales y virtuales de un mismo programa de Educación Superior, y de cómo interactúan diferentes factores que inciden en el logro del estudiante en ambas modalidades (Arias-Velandia, 2015).

2. Existe ya un conjunto importante de estudios que comparan programas presenciales y virtuales, o los efectos de un mismo curso presentado en modalidad presencial o virtual, pero es poco frecuente que los estudios planteen otros factores que pueden incidir en los logros más allá de la sola "presentación" (presencial o virtual) de los cursos (Arias-Velandia, Hederich \& Trujillo-Flórez, 2015).

3. También es poco frecuente que se planteen problemas sobre la forma de presentar evaluaciones o que planteen distintos formatos de evaluación o recolección de evidencias sobre el logro de los estudiantes (Arias-Velandia, Hederich \& Trujillo-Flórez, 2015). Esto puede presentar diferencias con respecto al logro evaluado externamente (mediante pruebas censales) o internamente (en evaluaciones confeccionadas por los profesores o tutores de los cursos), en las cuales Hederich y Camargo (2000) han mostrado diferencias importantes en estudiantes de quince años en la ciudad de Bogotá

Dado lo anterior, la pregunta que surge concretamente es: ¿Existen diferencias en el logro educativo de egresados de un programa (administración de empresas) en estudiantes de modalidad presencial y virtual de las instituciones colombianas de Educación Superior, que se ubiquen en promedios históricos cercanos en la prueba Saber Pro del ICFES?

\section{Método}

\section{Tipo de estudio y diseño}

Este es un estudio del alcance correlacional, ya que establece posibles asociaciones entre variables, sin que ninguna haya sido manipulada directa o intencionalmente por los investigadores (Hernández Sampieri, Fernández Collado y Baptista Lucio, 2010).

En este caso, estudia la asociación de las variables institución (Politécnico Grancolombiano, EAN) y modalidad (presencial, virtual), tomadas como independientes, sobre los puntajes obtenidos por los estudiantes en las pruebas Saber Pro que implementa el ICFES en Colombia, tomadas como dependientes (puntajes en subpruebas: lectura crítica, razonamiento cuantitativo, competencias ciudadanas e inglés).

El diseño que corresponde a esta investigación es cuasiexperimental porque no hay control completo de factores concernientes a los estudiantes en las instituciones y no fueron seleccionados completamente al azar, ya que se tomaron todos los correspondientes a la institución que presentaron examen Saber Pro en el periodo señalado.

Sin embargo, las variables independientes tienen un grado de control relativo por ser derivados del mismo examen Saber Pro de las bases de datos del ICFES de todos los estudiantes del país que presentaron dicha prueba (Hernández Sampieri, Fernández Collado y Baptista Lucio, 2010).

\section{Participantes}

Entre 194 estudiantes del programa Administración de Empresas de los cuales en la modalidad virtual se encuentran 29 estudiantes de la Institución Universitaria Politécnico Grancolombiano y 29 de la EAN; en la modalidad presencial se encuentran 68 estudiantes de la Institución Universitaria Politécnico Grancolombiano y 68 de la EAN. 


\section{Instrumentos}

Prueba Saber Pro: instrumento estandarizado para la evaluación externa de la calidad de la Educación Superior. Forma parte de un conjunto de instrumentos que el gobierno colombiano dispone para evaluar la calidad del servicio público educativo y ejercer su inspección y vigilancia (ICFES 2015). Saber Pro se compone de las siguientes subpruebas:

- Lectura crítica, que tiene contenidos de lectura, pensamiento crítico y entendimiento interpersonal, y evalúa el desempeño de leer analítica y reflexivamente la comprensión de los planteamientos expuestos en un texto y la identificación de sus perspectivas y juicios de valor e identificando y recuperando información presente en uno o varios textos, mediante la construcción del sentido global, el establecimiento de relaciones entre enunciados y la evaluación de intencionalidades.

- Razonamiento cuantitativo, que tiene contenidos relacionados con uso de lenguaje cuantitativo y solución de problemas; evalúan la comprensión de conceptos básicos de matemáticas para analizar, modelar y resolver problemas, aplicando métodos y procedimientos cuantitativos y esquemáticos y la interpretación de datos, la formulación de problemas y la ejecución, evaluación y validación de procedimientos y estrategias.

- Inglés, evalúa la comunicación efectiva en inglés; la subprueba permite clasificar a los evaluados en cuatro categorías de desempeño, según las bandas del Marco Común Europeo.

- Competencias ciudadanas y Entendimiento del entorno, permite reconocer y valorar la diversidad cultural y los derechos individuales y colectivos; entender, en el contexto nacional y global, los grandes problemas sociales, económicos, políticos y ambientales contemporáneos e identificar dilemas éticos en situaciones concretas 5 .

\section{Procedimiento}

Se accede a la base de datos del sistema FTP-ICFES del Sistema Nacional de Evaluación de la Educación (ICFES) con el fin de obtener los resultados de las pruebas Saber Pro 2012 del segundo semestre del año. Por medio de la herramienta SNIES del Ministerio de Educación, se realiza una depuración verificando cuáles universidades tienen el mismo programa en ambas modalidades en comparación con la Institución Universitaria Politécnico Grancolombiano.

Al depurar la base con la información de programa y universidad se realiza una importante delimitación de variables en las que se escogen únicamente los resultados de las subpruebas (anteriormente mencionadas) evaluadas por el ICFES.

\section{Análisis}

Para la realización del análisis correspondiente a este estudio se realizaron previamente análisis descriptivos de las variables estudiadas (media, mediana, desviación estándar y rango intercuartil) y análisis preliminar de gráficos de tendencia de asociación entre variables y coeficientes de correlación Rho de Spearman entre las mismas.

Uno de estos procesamientos previos incluyó la prueba para analizar el tipo de distribución que tenían los puntajes de las subpruebas del ICFES analizadas. Se usaron entonces las pruebas de normalidad Shapiro Wilk

5. Existe una subprueba adicional Escritura o Composición escrita, que evalúa la comunicación de ideas por escrito referidas a un tema. Los escritos se califican teniendo en cuenta la elaboración de la intención y el propósito, la claridad de la estructura, la organización y el manejo del lenguaje y las convenciones. Esta subprueba, sin embargo, no se analizó en el presente estudio, debido a la falta de datos de muchos de los estudiantes seleccionados en la prueba. 
Resultados en Saber Pro de estudiantes de modalidad presencial y virtual en dos universidades colombianas

en los grupos conformados con menos de 50 personas $^{6}$ (modalidad virtual en ambas instituciones, con 29 sujetos en cada universidad) y Kolmogorov Smirnoff en los grupos conformados con más de 50 personas $^{7}$ (modalidad presencial en ambas instituciones, con 68 sujetos en cada universidad).

Adicionalmente, se realiza la prueba no paramétrica U de Mann-Whitney de comparación de muestras independientes entre estudiantes de modalidad presencial y modalidad virtual en general, y análisis de varianza (Anova) para los resultados en subprueba de lectura crítica y análisis de Kruskall-Wallis (análisis de varianza no paramétrico) en subpruebas de razonamiento cuantitativo, competencias ciudadanas e inglés comparando el efecto en dichos puntajes tanto de modalidad (presencial o virtual) como de institución (Politécnico o EAN). En todos los análisis se tomó como nivel de significancia $\mathrm{P} \leq 0.05$

\section{Resultados}

\section{Pruebas de normalidad}

Se realizó una prueba de normalidad de Shapiro Wilk para todas las variables de modalidad virtual (tabla 1). Para la variable de modalidad presencial se aplicó la prueba de normalidad de Kolmogorov Smirnoff.

De estos grupos, resultaron con distribución no paramétrica Competencias ciudadanas en la EAN en modalidad Virtual, Razonamiento numérico en la Institución Universitaria Politécnico Grancolombiano en la modalidad presencial e inglés puntaje en ambas universidades y ambas modalidades.

\section{Comparaciones entre variables}

En la tabla 2 se muestra la comparación entre pruebas no paramétricas para muestras independientes y las subpruebas Competencias ciudadanas, Razonamiento numérico, Lectura crítica e Inglés puntaje de ambas

\begin{tabular}{|c|c|c|c|c|c|c|c|}
\hline \multicolumn{8}{|c|}{ Prueba de Normalidad Kolmogorov-Smirnov y Shapiro-Wilk } \\
\hline & \multirow[t]{2}{*}{ Universidad y modalidad } & \multicolumn{3}{|c|}{ Kolmogorov-Smirnov ${ }^{\mathrm{a}}$} & \multicolumn{3}{|c|}{ Shapiro-Wilk } \\
\hline & & Statistics & df & Sig. & Statistics & df & Sig. \\
\hline \multirow{4}{*}{$\begin{array}{l}\text { Competencias } \\
\text { ciudadanas }\end{array}$} & Politécnico Grancolombiano virtual & .129 & 29 & $.200^{*}$ & .963 & 29 & .384 \\
\hline & \begin{tabular}{|ll}
$\begin{array}{l}\text { Politécnico } \\
\text { presencial }\end{array}$ & Grancolombiano \\
\end{tabular} & .088 & 68 & $.200^{*}$ & .974 & 68 & .156 \\
\hline & EAN virtual & .175 & 29 & .024 & .894 & 29 & .007 \\
\hline & EAN presencial & .092 & 68 & $.200^{*}$ & .974 & 68 & .157 \\
\hline \multirow{4}{*}{$\begin{array}{l}\text { Razonamiento } \\
\text { numérico }\end{array}$} & Politécnico Grancolombiano virtual & 150 & 29 & .096 & .942 & 29 & .113 \\
\hline & \begin{tabular}{|ll}
$\begin{array}{l}\text { Politécnico } \\
\text { presencial }\end{array}$ & Grancolombiano \\
\end{tabular} & 116 & 68 & .023 & .977 & 68 & .234 \\
\hline & EAN virtual & .148 & 29 & .104 & .951 & 29 & .193 \\
\hline & EAN presencial & .093 & 68 & $.200^{*}$ & .986 & 68 & .627 \\
\hline \multirow[t]{4}{*}{ Lectura crítica } & Politécnico Grancolombiano virtual & .099 & 29 & $.200^{*}$ & .976 & 29 & .735 \\
\hline & Grancolombiano & 100 & 68 & .090 & .970 & 68 & .098 \\
\hline & EAN virtual & .116 & 29 & $.200^{*}$ & .985 & 29 & .936 \\
\hline & EAN presencial & .083 & 68 & $.200^{*}$ & .984 & 68 & .512 \\
\hline \multirow[t]{4}{*}{ Inglés puntaje } & Politécnico Grancolombiano virtual & .296 & 29 & .000 & .594 & 29 & .000 \\
\hline & \begin{tabular}{|l|} 
Politécnico \\
presencial
\end{tabular} & .232 & 68 & .000 & .863 & 68 & .000 \\
\hline & EAN virtual & .239 & 29 & .000 & .828 & 29 & .000 \\
\hline & EAN presencial & .161 & 68 & .000 & .904 & 68 & .000 \\
\hline
\end{tabular}

Tabla 1. Prueba de normalidad Kolmogorov-Smirnov y Shapiro-Wilk en variables Universidad, Modalidad, Competencias ciudadanas, Razonamiento numérico, Lectura crítica e Inglés puntaje

Fuente: datos propios procesados en IBM SPSS Statistics 17.

universidades comparando modalidades presencial y virtual. Se muestra la existencia de diferencias significativas en la variable Inglés puntaje.

\begin{tabular}{|c|l|l|l|l|}
\hline \multicolumn{5}{|c|}{ Prueba no paramétrica para muestras independientes } \\
\hline & $\begin{array}{c}\text { Competencias } \\
\text { ciudadanas }\end{array}$ & $\begin{array}{c}\text { Razonamiento } \\
\text { numérico }\end{array}$ & $\begin{array}{c}\text { Lectura } \\
\text { crítica }\end{array}$ & $\begin{array}{c}\text { Inglés } \\
\text { puntaje }\end{array}$ \\
\hline $\begin{array}{c}\text { Mann- } \\
\text { Whitney U }\end{array}$ & 3644.500 & 3192.500 & 3811.500 & 2933.000 \\
\hline Z & -.838 & -2.103 & -.371 & -2.828 \\
\hline Asymp. Sig. & .402 & .036 & .711 & .005 \\
\hline
\end{tabular}

Tabla 2. Tabla de prueba no paramétrica para muestras independientes (U de Mann Whitney) de la modalidad presencial y virtual con las variables Competencias ciudadanas, Razonamiento numérico, Lectura crítica e Inglés puntaje

Fuente: datos propios procesados en IBM SPSS Statistics 17.

6. Shapiro Wilk: prueba usada para contrastar la normalidad de un conjunto de datos. Cuanto mayor sea este estadístico mayor desacuerdo habrá con la recta de normalidad, por lo que podremos rechazar la hipótesis nula; se usa para muestras menores a 50 datos (Shapiro, S.; Wilk, M, 1965).

7. Kolmogorov-Smirnov: prueba no paramétrica que permite medir el grado de concordancia existente entre la distribución de un conjunto de datos y una distribución teórica específica; usada para muestras mayores a 50 datos (García, R; González, J; Jornet, M, 2010). 
Deisy Ortiz Romero, Erika Gómez Villarreal \& Nicolás Arias Velandia

Los datos de la tabla 3 muestran que no existen diferencias significativas entre universidades y sus modalidades presenciales y virtuales en lectura crítica.

\begin{tabular}{|c|c|c|c|c|c|c|}
\hline \multicolumn{7}{|c|}{ Prueba paramétrica Anova } \\
\hline \multirow[b]{2}{*}{$\begin{array}{l}\text { (I) Universidad y } \\
\text { Modalidad }\end{array}$} & \multirow[b]{2}{*}{$\begin{array}{l}\text { (J) Universidad y } \\
\text { Modalidad }\end{array}$} & \multirow{2}{*}{$\begin{array}{c}\text { Mean } \\
\text { Difference } \\
\text { (I-J) }\end{array}$} & \multirow[b]{2}{*}{ Std. Error } & \multirow[b]{2}{*}{ Sig. } & \multirow[b]{2}{*}{$\begin{array}{l}\text { Lower } \\
\text { Bound }\end{array}$} & \multirow[b]{2}{*}{$\begin{array}{l}\text { Upper } \\
\text { Bound }\end{array}$} \\
\hline & & & & & & \\
\hline $\begin{array}{c}\text { Politécnico } \\
\text { Grancolombiano } \\
\text { virtual }\end{array}$ & $\begin{array}{c}\text { Politécnico } \\
\text { Grancolombiano } \\
\text { presencial }\end{array}$ & .2562 & .1760 & .466 & -200 & .712 \\
\hline \multirow{3}{*}{$\begin{array}{c}\text { Politécnico } \\
\text { Grancolombiano } \\
\text { virtual }\end{array}$} & EAN virtual & .2793 & .2084 & .539 & -.261 & .820 \\
\hline & & & & & & \\
\hline & EAN presencial & -.0452 & .1760 & .994 & -.501 & .411 \\
\hline $\begin{array}{c}\text { Politécnico } \\
\text { Grancolombiano } \\
\text { presencial }\end{array}$ & $\begin{array}{c}\text { Politécnico } \\
\text { Grancolombiano } \\
\text { virtual }\end{array}$ & -.2562 & .1760 & .466 & -.712 & .200 \\
\hline \multirow{2}{*}{$\begin{array}{c}\text { Politécnico } \\
\text { Grancolombiano } \\
\text { presencial }\end{array}$} & EAN virtual & .0231 & .1760 & .999 & -.433 & .479 \\
\hline & EAN presencial & -3015 & .1361 & .123 & -.654 & .051 \\
\hline EAN virtual & $\begin{array}{c}\text { Politécnico } \\
\text { Grancolombiano } \\
\text { virtual }\end{array}$ & -.2793 & .2084 & .539 & -.820 & .261 \\
\hline \multirow[t]{3}{*}{$\begin{array}{c}\text { EAN virtual } \\
\text { EAN presencial }\end{array}$} & $\begin{array}{c}\text { Politécnico } \\
\text { Grancolombiano } \\
\text { presencial }\end{array}$ & -.0231 & .1760 & .999 & -.479 & .433 \\
\hline & EAN presencial & -.3245 & .1760 & .256 & -.781 & .132 \\
\hline & $\begin{array}{c}\text { Politécnico } \\
\text { Grancolombiano } \\
\text { virtual }\end{array}$ & .0452 & .1760 & .994 & -.411 & .501 \\
\hline \multirow[t]{2}{*}{ EAN presencial } & $\begin{array}{l}\text { Politécnico } \\
\text { Grancolombiano } \\
\text { presencial }\end{array}$ & .3015 & .1361 & .123 & -.051 & .654 \\
\hline & EAN virtual & .3245 & .1760 & .256 & -.132 & .781 \\
\hline
\end{tabular}

Tabla 3. Prueba paramétrica para comparación de grupos (Anova) usando la variable Lectura crítica y las variables Universidad y Modalidad puntaje

Fuente: datos propios procesados en IBM SPSS Statistics 17.

En la tabla 4 se comparan los efectos de universidad y modalidad sobre las puntuaciones en Competencias ciudadanas, Razonamiento numérico e Inglés puntaje. Este resultado muestra puntaje bajo Inglés puntaje en la Institución Universitaria Politécnico Grancolombiano - modalidad virtual, puntajes medios en la Institución Universitaria Politécnico Grancolombiano - modalidad presencial y puntajes altos en la EAN en ambas modalidades. Del mismo modo, se encuentran tendencias a puntuaciones altas en ambas universidades en Competencias ciudadanas en modalidad virtual y tendencias a puntuaciones bajas en ambas universidades en razonamiento numérico en modalidad virtual.

\begin{tabular}{|c|c|c|c|}
\hline \multicolumn{4}{|c|}{ Prueba no paramétrica Kruskal-Wallis } \\
\hline & Universidad y modalidad & $\mathrm{N}$ & Mean Rank \\
\hline \multirow{5}{*}{$\begin{array}{l}\text { Competencias } \\
\text { ciudadanas }\end{array}$} & Politécnico Grancolombiano virtual & 29 & 103.55 \\
\hline & Politécnico Grancolombiano presencial & 68 & 89.74 \\
\hline & EAN virtual & 29 & 101.78 \\
\hline & EAN presencial & 68 & 100.86 \\
\hline & Total & 194 & \\
\hline \multirow{5}{*}{$\begin{array}{l}\text { Razonamiento } \\
\text { numérico }\end{array}$} & Politécnico Grancolombiano virtual & 29 & 76.41 \\
\hline & Politécnico Grancolombiano presencial & 68 & 101.04 \\
\hline & EAN virtual & 29 & 92.67 \\
\hline & EAN presencial & 68 & 105.01 \\
\hline & Total & 194 & \\
\hline \multirow[t]{5}{*}{ Inglés puntaje } & Politécnico Grancolombiano virtual & 29 & 55.05 \\
\hline & Politécnico Grancolombiano presencial & 68 & 98.51 \\
\hline & EAN virtual & 29 & 105.09 \\
\hline & EAN presencial & 68 & 111.35 \\
\hline & Total & 194 & \\
\hline
\end{tabular}

Tabla 4. Prueba no paramétrica Kruskal-Wallis para las variables Competencias Ciudadanas, Razonamiento Numérico e Inglés Puntaje en comparación con Universidad y Modalidad

Fuente: datos propios procesados en IBM SPSS Statistics 17.

En la tabla 5 se evidencia que el efecto de universidad y modalidad sobre puntuaciones es significativo en la variable Inglés puntaje.

\begin{tabular}{|l|l|l|l|}
\hline \multicolumn{4}{|c|}{ Tabla Estadísticos Kruskal-Wallis } \\
\hline & $\begin{array}{c}\text { Competencias } \\
\text { ciudadanas }\end{array}$ & $\begin{array}{c}\text { Razonamiento } \\
\text { numérico }\end{array}$ & $\begin{array}{c}\text { Inglés } \\
\text { puntaje }\end{array}$ \\
\hline Chi-Cuadrado & 2.055 & 5.812 & 21.323 \\
\hline Df & 3 & 3 & 3 \\
\hline Asymp. Sig. & .561 & .121 & .000 \\
\hline \multicolumn{4}{|l}{} \\
\hline
\end{tabular}

Tabla 5. Tabla de estadísticos generales Kruskal-Wallis para las variables Competencias ciudadanas, Razonamiento numérico e Inglés puntaje Fuente: datos propios procesados en IBM SPSS Statistics 17.

De los resultados se infiere que la variable Inglés puntaje presenta diferencias significativas entre ambas universidades y ambas modalidades en donde los puntajes más bajos se evidencian en la Institución Universitaria Politécnico Grancolombiano en modalidad Virtual y puntajes medios en modalidad presencial a comparación con la EAN que tiene altos puntajes en ambas modalidades.

El análisis de resultados muestra que no existe ninguna diferencia significativa entre las demás variables aunque sí se demuestran tendencias de puntuaciones altas en ambas universidades en la variable Competencias ciudadanas en modalidad virtual y tendencias a puntuaciones bajas 
en ambas universidades en la variable Razonamiento cuantitativo en modalidad virtual.

\section{Discusión y conclusiones}

El análisis de los datos muestra que existen diferencias entre los resultados del Saber Pro. Se encuentra una diferencia significativa entre el puntaje de las instituciones educativas y no entre la modalidad, aunque se evidencian tendencias altas en la modalidad virtual en el subgrupo Competencias ciudadanas y tendencias bajas en la modalidad virtual en el subgrupo Razonamiento cuantitativo.

Sin embargo, no se encuentran diferencias estadísticamente significativas en Lectura crítica y en Competencias ciudadanas; la ausencia de diferencias significativas entre modalidades es la tendencia que con mayor frecuencia se reporta en las investigaciones pues cerca de $66 \%$ no muestra diferencias entre los estudiantes de ambas modalidades aunque en la mayoría de investigaciones no se plantean pruebas censales a modo de criterio de evaluación externa como es el Saber Pro, cuyo hallazgo es planteado por Arias-Velandia, Hederich y TrujilloFlórez (2015).

Adicionalmente, se encuentra que en los subgrupos de Inglés puntaje existen diferencias estadísticamente significativas entre las modalidades y entre las instituciones. Para la EAN existe una ubicación alta en modalidad virtual y modalidad presencial, para la Institución Universitaria Politécnico Grancolombiano existe una ubicación media en modalidad presencial y una ubicación baja en modalidad virtual. Lo planteado por Rodríguez Albor, Gómez Lorduy y Ariza Dau (2014) habla sobre los efectos donde no es posible separar la modalidad (presencial o virtual) de otras variables como el modelo pedagógico que parecía diferir entre modalidades, tal como se evidencia en los resultados de la presente investigación.

Otro hallazgo que se encuentra en el estudio es que para la subprueba Razonamiento cuantitativo no se muestran diferencias estadísticamente significativas entre instituciones o modalidades, pero se encuentra una tendencia a registrar puntajes más bajos en la Institución Universitaria Politécnico Grancolombiano virtual y la EAN virtual. Estas tendencias de evaluación del logro poco concentradas en las medidas externas se podrían atribuir a que en la modalidad presencial se presentan elementos de interacción social y escucha lo que favorece un poco más a los estudiantes que valoran estos elementos (López, 2010; López \& Triana, 2013; Smith et al., 2001).

Las tendencias en la subprueba Competencias ciudadanas y Razonamiento cuantitativo resultan interesantes pero debido a su falta de significancia no son concluyentes, por ello se sugiere la continuación de este estudio con una muestra mayor, así mismo tema importante por tratar a los investigadores es si existen diferencias entre los modelos pedagógicos, entre las modalidades o entre las instituciones que puedan generar diferentes efectos en los resultados de los estudiantes.

\section{Referencias}

Abuchar, A. \& Simanca, H. (2013). E-Learning en procesos de evaluación académica; pruebas Saber Pro, recuperado de: http://comunidad.udistrital. edu.co/revistavinculos/files/2013/09/e-learningen-procesos-de-evaluaci $\% \mathrm{C} 3 \% \mathrm{~B} 3 n$-academicaPruebas-Saber-Pro.pdf

Arias-Velandia, N. (2015). Logro educativo comparado entre Educación Superior presencial y virtual en Colombia: algunas tendencias y grandes desafíos. Texto presentado como ponencia en el Congreso Iberoamericano de Educación Virtual, Pontificia Universidad Javeriana, Bogotá, Noviembre de 2015. Disponible en el Repositorio Institucional Alejandría, Institución Universitaria Politécnico Grancolombiano, en: http://repository. poligran.edu.co/bitstream/10823/695/1/ E D U C A C I O N \% 20 S U P E R I O R \% 20 PRESENCIAL\%20Y\%20VIRTUAL.pdf

Arias-Velandia, N., Hederich, C. \& Guarnizo, J. (2014). Aspectos para la comparación del logro académico de estudiantes de modalidad presencial y virtual en instituciones colombianas de Educación Superior. 
En Sociedad Española de Pedagogía, Universidad Católica Silva Henríquez y Universidad Católica de Valparaíso (ed.). Memorias VI Congreso Iberoamericano de Pedagogía. Santiago, Chile: Universidad Católica Silva Henríquez.

Arias-Velandia, N., Hederich, C. \& Trujillo-Flórez, L. M. (2015). Avances en me meta-análisis del logro comparado entre Educación Superior presencial y virtual: tendencias y tipos de evaluación. Memorias del Seminario Internacional Virtual Educa, México, 2015.

Brownstein, B.; Brownstein, D.; Gerlowski, D. (2008). Web-Based vs. Face-To-Face MBA Classes: A comparative assessment study. Journal of College Teaching \& Learning, 5(11), 41-48.

Camargo,A.(2013). Conferencistas versus conversadores. Estilos de enseñanza de profesores de ciencias y su relación con el estilo cognitivo. Revista Colombiana de Educación, 64, 273 - 307.

Camargo, A. \& Hederich, C. (2007). El estilo de comunicación y su presencia en el aula de clase. Folios, 26, 3 - 12.

Carrol, N. E. \& Burke, M. (2010). Learning Effectiveness Using Different Teaching Modalities. American Journal of Business Education, 3(12), 65-76.

Coma del C., M.J; Cordero, J.; Abáigar, P.; Peña, H.J.; Mateos, J.J. (2006). Usefulness of an InternetBased Thematic Learning Network: Comparison of Effectiveness with Traditional Teaching. Medical Informatics and the Internet in Medicine, 31(1), 59-66.

García, R.; González, J. y Jornet, J. (2010). SPSS: Pruebas no paramétricas: Kolmogorov Smirnov. Grupo de Innovación Educativa, Innova mide. Recuperado el 1 de Junio de 2015, de http://www. uv.es/innomide/spss/SPSS/SPSS_0802A.pdf
Gibson, J. W. (2008). A Comparison Of Student Outcomes And Student Satisfaction In Three MBA Human Resource Management Classes Based On Traditional Vs. Online Learning. Journal of College Teaching \& Learning, 5(8), 1-9.

Hederich, C. \& Camargo, A. (2000). Estilo cognitivo y logro educativo en la ciudad de Bogotá D. C. Bogotá: Instituto para la Investigación Educativa y el Desarrollo Pedagógico, IDEP / Centro de Investigaciones de la Universidad Pedagógica Nacional, CIUP.

Hernández Sampieri, R., Fernández Collado, C. \& Baptista Lucio, P. (2010). Metodología de la investigación, 6ta edición. México: McGrawHill.

Kirtman, L. (2009). Online Vs. In-Class Courses: An Examination of Differences in Learning Outcomes. Issues in Teacher Education, 18(2), 103-116.

Lapsley, R., Kulik, B., Moody, R. \& Arbaught, B. (2008). Is Identical Really Identical? An Investigation of Equivalency Theory and Online Learning. The Journal of Education Online. 5(1), 1 -19.

Larson, D. K. \& Sung, C. H. (2009). Comparing Student Performance: Online Versus Blended Versus Face-To-Face. Journal of Asynchronous Learning Networks, 13(1), 31-42.

LeBrance, G. (2003). Blended learning: The convergence of e-learning and meetings. Washington: International Franchise Association.

López, O. (2010). Aprendizaje autorregulado, estilo cognitivo y logro académico en ambientes computacionales. Tesis no publicada para optar al título de Doctor (Ph.D.) en Educación, Universidad Pedagógica Nacional, Bogotá, Colombia. 
López, O. \& Triana, S. (2013). Efecto de un activador computacional de autoeficacia sobre el logro de aprendizaje en estudiantes de diferente estilo cognitivo. Revista Colombiana de Educación, 64, $225-244$.

Lugo, M. T., Vera-Rossi, M. \& Flood, C. (2004). Educación Superior virtual en Argentina: un relevamiento necesario. En Unesco (ed.), La Educación Superior virtual en América Latina y el Caribe (pp. 51 - 84). Caracas: Unesco.

McGovern, G. (2004). Teaching Online vs. Face-to-Face. Newsletter of the American Library Association Continuing Library Education Network \& Exchange Round Table. 20(4), 1-8.

Molina, A., Wiley, E., \& Van, S. (2013). Icfes Mejor Saber. Valor agregado Educación Superior en Colombia. Recuperado el 7 de Noviembre de 2013, de http://www.icfes.gov.co/seminario/programaacademico.html? start $=5$

Moreno (1998). El desarrollo de habilidades como objetivo educativo. Una aproximación conceptual. Educar, Julio - Septiembre.

Onrubia, J. (2005). Aprender y enseñar en entornos virtuales: actividad conjunta, ayuda pedagógica y construcción del conocimiento. RED. Revista de Educación a Distancia, número monográfico II. Consultado el 9 de Febrero de 2005 en http:// www.um.es/ead/red/M2/

Padilla Omiste, A. (2004). Diagnóstico de la Educación Superior virtual en Bolivia. En Unesco (ed.), La Educación Superior virtual en América Latina y el Caribe (pp. 85 - 112). Caracas: Unesco.

Prensky, M. (2001). Nativos e Inmigrantes digitales. Adaptación al castellano del texto original "Digital Natives, Digital Immigrants". Distribuidora SEK, S.A. 2010.
Rivera Rivera, K. (2004). La Educación Superior a distancia en Centroamérica. En Unesco (ed.), La Educación Superior virtual en América Latina y el Caribe (pp. 145 - 164). Caracas: Unesco.

Rodríguez Albor, G., Gómez Lorduy, V. \& Ariza Dau, M. (2014). Calidad de la Educación Superior a distancia y virtual: un análisis de desempeño académico en Colombia. Investigación \& Desarrollo, 22(1), $79-121$.

Silvio, J. (2004). Tendencias de la Educación Superior virtual en América Latina y el Caribe. En Unesco (ed.), La Educación Superior virtual en América Latina y el Caribe (pp. 5 -28). Caracas: Unesco.

Shapiro, S. S. \& Wilk, M. B. (1965). An analysis of variance test for normality (complete samples). Biometrika, 52 (3-4) pp. 591-611

Shutte, J. (1997). Virtual Teaching in Higher Education: The New Intellectual Super-Highway or Just Another Traffic Jam? Consultado el 16/04/2013 en: http://ddi.cs.uni-potsdam.de/HyFISCH/ Teleteaching/VirtualTeachingSchutte.htm

Simonson, M., Schlosser, C. \& Hanson, D. (1999). Theory and distance education: a new discussion. The American Journal of Distance Education, 13(1), $60-75$.

Smith, G.G.; Ferguson, D.L. \& Caris, A. (2001). Teaching College Courses Online vs Face-to-Face. The Journal Digital Edition. Revisado el 16/04/2013 en: http://thejournal.com/Articles/2001/04/01/ Teaching-College-Courses-Online-vs-FacetoFace.aspx

Suárez, J. \& Anaya, N. (2004). Educación a distancia y presencial: diferencias en los dos componentes cognitivo y motivacional de estudiantes universitarios. Revista Iberoamericana de Educación a Distancia RIED, 7(1-2), 65-75. 
Turpo, O. (2012). Desarrollo y perspectiva de la modalidad educativa Blended Learning en las universidades de Iberoamérica. Educar, 1(48), 123 - 147.

Tutty, J. I. \& Kleine, J. D. (2008). Computer-mediated Instruction: A Comparison of Online and Face-toFace Collaboration. Education Technology and Research Development (56), 101-124.

Vianney, J., Torres, P. \& Farias, E. (2004). La Educación Superior a distancia en Brasil. En Unesco (ed.), La Educación Superior virtual en América Latina y el Caribe (pp. 113 - 144). Caracas: Unesco.

Wong, L. \& Tantall, A. (2009). The Need to Balance the Blend: Online Versus Face-to-Face Teaching in an Introductory Accounting Subject. Issues in Informing Science and Information Technology, 6, 309-322. 